\title{
Variación sociofonética: comparación de análisis graduales y categóricos*
}

\author{
FERNANDO MELERO GARCÍA**
}

Recepción: 03 de diciembre de 2020

Aprobación: 18 de febrero de 2021

Forma de citar este artículo: Melero, F. (2021). Variación sociofonética: comparación de análisis graduales y categóricos. Cuadernos de Lingüística Hispánica, (37), e12184

J01 https://10.19053/0121053X.n37.2021.12184

\footnotetext{
* Artículo de investigación.

** Doctor en Lingüística Hispánica por la Universidad de Indiana. Profesor e investigador en el Departamento de Lenguas Modernas de la Universidad de Texas en Arlington. fernando.melerogarcia@uta.edu @ orcid.org/0000-0002-3554-314X.
} 


\section{Resumen}

En este artículo se comparan los resultados de dos modelos de análisis sociofonético diferentes: un modelo continuo y uno categórico. La variable sociofonética investigada es la producción de /d/ intervocálica en Madrid. En el modelo continuo, el debilitamiento de /d/ fue calculado tomando medidas acústicas de intensidad, de tal forma que dicho análisis puede dar cuenta de manera precisa de todo el espectro de posibles realizaciones de /d/ (i.e., desde muy oclusivas hasta muy debilitadas). En el modelo categórico, el debilitamiento de /d/ fue codificado de manera binaria (retención o elisión total) de modo que este análisis informa únicamente del caso más extremo de debilitamiento consonántico: la elisión. En ambos análisis estadísticos se incluyeron las mismas variables independientes lingüísticas y extralingüísticas. Aunque los resultados de ambos análisis muestran semejanzas significativas en cuanto al efecto de las variables independientes, también se encontraron diferencias. Además de proveer una caracterización sociofonética de la producción de /d/ en Madrid, en este artículo se resaltan algunas consideraciones que se deben tener en cuenta al examinar fenómenos sociofonéticos con métodos tanto continuos como categóricos.

Palabras clave: acústica, fonética, metodología, sociolingüística.

\section{Sociophonetic Variation: Comparison of Gradual and Categorical Analysis}

\section{Abstract}

This paper compares results of two different sociophonetic analysis models: a continuous model and a categorical one. The sociophonetic variable investigated is the production of intervocalic / $\mathrm{d} /$ in Madrid. In the continuous model, the weakening of /d/ was calculated by taking acoustic intensity measurements, in such a way that said analysis can accurately inform on the entire spectrum of possible productions of /d/ (i.e., from very occlusive to very weakened). In the categorical model, the weakening of / $\mathrm{d} /$ was coded in a binary way (retention or total elision) so that this analysis only informs of the most extreme case of consonant weakening: elision. The same linguistic and extralinguistic independent variables were included in both statistical analyses. Although the results of both analyses show significant similarities regarding the effect of the independent variables, differences were also found. In addition to providing a sociophonetic characterization of the production of $/ \mathrm{d} /$ in Madrid, this article highlights some considerations that must be taken into account when examining sociophonetic phenomena with both continuous and categorical methods.

Keywords: acoustics, phonetics, methodology, sociolinguistics. 


\section{Variation socio-phonétique: comparaison d'analyses graduelles et catégorielles}

\section{Résumé:}

Cet article compare les résultats de deux modèles différents d'analyse sociophonique: un modèle continu et un modèle catégoriel. La variable socio-phonique étudiée est la production du / $\mathrm{d} /$ intervocalique à Madrid. Dans le modèle continu, l'affaiblissement de / d / a été calculé en prenant des mesures acoustiques de l'intensité, de sorte qu'une telle analyse puisse rendre compte avec précision de tout le spectre des réalisations possibles de / $\mathrm{d} /$ (c'est-à-dire de très occlusif à très affaibli). Dans le modèle catégoriel, l'affaiblissement de / $\mathrm{d} / \mathrm{a}$ été codé de manière binaire (rétention ou élision totale) afin que cette analyse ne prenne en compte que le cas le plus extrême d'affaiblissement consonantique: l'élision. Les mêmes variables indépendantes linguistiques et extralinguistiques ont été incluses dans les deux analyses statistiques. Bien que les résultats des deux analyses montrent des similitudes significatives en termes d'effet des variables indépendantes, des différences ont également été constatées. En plus de fournir une caractérisation socio-phonique de la production du / d / à Madrid, cet article met en évidence certaines considérations a prendre lors de l'examen des phénomènes socio-phoniques avec des méthodes à la fois continues et catégorielles.

Mots clés: acoustique, phonétique, méthodologie, sociolinguistique.

Variação sociofonética: comparação da análise gradual e categorica

\section{Resumo}

Este artigo compara os resultados de dois modelos diferentes de análise sócio fonética: um modelo contínuo e um modelo categórico. A variável sócio fonética investigada é a produção do / d / intervocálico em Madrid. No modelo contínuo, o enfraquecimento de / d / foi calculado tomando medidas de intensidade acústica, de tal forma que a referida análise possa contabilizar com precisão todo o espectro de possíveis realizações de / $\mathrm{d} /$ (ou seja, de muito oclusivo a muito enfraquecido). No modelo categórico, o enfraquecimento de / $\mathrm{d} /$ foi codificado de forma binária (retenção ou elisão total), de forma que esta análise apenas explica 0 caso mais extremo de enfraquecimento consonantal: a elisão. As mesmas variáveis independentes linguísticas e extralinguísticas foram incluídas em ambas as análises estatísticas. Embora os resultados de ambas as análises mostrem semelhanças significativas quanto ao efeito das variáveis independentes, também foram encontradas diferenças. Além de fornecer uma caracterização sociofônica da produção de / d / em Madrid, este artigo destaca algumas considerações que devem ser levadas em conta ao examinar os fenômenos sociofônicos com métodos contínuos e categóricos.

Palavras-chave: acústica, fonética, metodologia, sociolinguística. 


\section{Introducción}

Los fonemas / $\mathrm{b} \mathrm{d} \mathrm{g/} \mathrm{del} \mathrm{español} \mathrm{se} \mathrm{producen} \mathrm{con} \mathrm{diferentes} \mathrm{grados} \mathrm{de}$ constricción en diferentes contextos. Una idea ampliamente aceptada sobre estos fonemas es que en la mayoría de sus contextos se producen sin un cierre completo, es decir, como aproximantes (Hualde, 2014). Este tipo de debilitamiento consonántico, comúnmente conocido como espirantización, ha llamado la atención de numerosos investigadores debido precisamente a su alta frecuencia en español (Lozano, 1979; Mascaró, 1991; Ortega-Llevaria, 2003; Rogers, 2016). Específicamente, uno de los fenómenos que ha despertado el interés de numerosos investigadores es el debilitamiento o elisión de /d/ en posición intervocálica (Gómez \& Gómez, 2010; Jiménez, 2020; Malaver \& Perdomo, 2016; Moya, 2009). Antes de que los programas informáticos de análisis acústico estuvieran tan disponibles como lo están hoy en día, muchos estudios sobre el debilitamiento de /d/ analizaron este fenómeno de una forma categórica. Es decir, el debilitamiento de /d/ se codificaba de manera auditiva y mediante la creación de categorías finitas. Generalmente, dichas categorías incluían dos opciones: la retención de /d/ o su elisión. Sin embargo, algunos investigadores (Carrasco et al., 2012; Díaz-Campos, 2016; Díaz-Campos et al., 2015; File-Muriel \& Brown, 2011) han señalado en los últimos años el hecho de que el debilitamiento consonántico no es categórico por naturaleza sino gradual y, por tanto, los métodos usados para su investigación deberían reflejar dicha gradualidad. Basándonos en estas ideas, el propósito de este artículo es llevar a cabo una investigación sociolingüística en la que se examine el debilitamiento de /d/ intervocálica usando dos metodologías diferentes: un análisis categórico y uno continuo, para comprender las ventajas 0 limitaciones de los diferentes tipos de análisis.

\section{Estudios previos}

\section{Debilitamiento de /d/ intervocálica en España}

Los análisis sociolingüísticos sobre la /d/ intervocálica en España han demostrado que el grado de debilitamiento de este sonido en dicho país varía enormemente de una región a otra (Samper, 2011). Por ejemplo, se ha encontrado que las tasas de elisión de /d/ intervocálica en Andalucía oriental son muchos más altas que en otras regiones. Moya (1979) y Gómez (1994) reportan unas tasas de elisión del $65 \%$ y $78 \%$ en dos localidades diferentes de Andalucía oriental, mientras que Pérez (2003) y Blanco (2004) reportan tasas de elisión significativamente más bajas en El Hierro (15\%) y Alcalá de Henares (18\%). Para la variedad que se estudia en este artículo se ha reportado una tasa de elisión del 21 \% (Gil, 2004), lo cual significa que la gran mayoría de casos de /d/ intervocálica se retiene en esta variedad. Además de la región geográfica, se ha demostrado que factores tanto lingüísticos como extralingüísticos condicionan significativamente el debilitamiento de /d/.

Con respecto a los factores lingüísticos, un hallazgo común en múltiples variedades es que los participios pasados favorecen significativamente la elisión 
de /d/ intervocálica y, más específicamente, la elisión suele ser más frecuente en participios pasados que terminan en -ado (Samper, 2011). Con respecto al contexto fonético, se observan tendencias similares en diferentes variedades peninsulares: cuando la palabra que contiene la $/ \mathrm{d} /$ no es un participio pasado, las vocales $/ 0 / \mathrm{y}$ /a/ generalmente favorecen la elisión, mientras que las vocales /i/ y /u/ favorecen la retención. Finalmente, también se ha reportado un efecto significativo de la categoría gramatical de la palabra que contiene la /d/: se ha encontrado que hay más elisión en adjetivos que en sustantivos (Samper, 2011).

En lo que se refiere a los factores extralingüísticos, se ha examinado en estudios previos el rol de variables como el género, la edad o el nivel socioeconómico del hablante. Mientras que el efecto de los factores lingüísticos previamente mencionados suele ser consistente en diferentes variedades, los factores sociales muestran más variación cuando comparamos diferentes regiones. Por ejemplo, en diferentes regiones de España, los hombres suelen elidir la /d/ intervocálica más frecuentemente que las mujeres, excepto en El Hierro, donde no se observó ningún efecto significativo del género del hablante (Samper, 2011). Con respecto a la edad, los resultados son más diversos aún: mientras que las generaciones más jóvenes favorecen la retención en Gran Canaria y Getafe, lo opuesto es cierto para Málaga y Granada. Finalmente, en general, también se ha encontrado que la tasa de elisión de /d/ disminuye a medida que el nivel socioeconómico del hablante aumenta (Samper, 2011).

Si bien es cierto que estos resultados ofrecen una caracterización relevante sobre las diferentes variedades del español y nos permiten hacer algunas comparaciones entre variedades, no aportan una caracterización precisa o detallada de la producción del nivel de debilitamiento de la /d/ intervocálica. Por ejemplo, cuando hablamos de retención de /d/, dicha retención puede ser una producción muy debilitada o reforzada. Es decir, existe un continuo de posibles realizaciones de /d/ que pasa desapercibido en los estudios que emplean métodos categóricos. En la siguiente sección se ofrece un resumen de estudios recientes que han examinado fenómenos de debilitamiento consonántico de una manera continua a través del análisis de pistas acústicas concretas.

\section{La naturaleza continua del debilitamiento consonántico}

Algunos estudios previos que destacan la naturaleza continua del debilitamiento consonántico incluyen los de File-Muriel y Brown (2011), Carrasco et al. (2012), y Hualde et al. (2011). Por un lado, File-Muriel y Brown (2011) llevan a cabo un análisis continuo del debilitamiento de /s/ en el español caleño. Dejando atrás la categorización tradicional de /s/ como [s], [h] o Ø, estos autores tomaron medidas acústicas continuas de este sonido, tales como la duración de la /s/, el centro de gravedad (una medida acústica sobre la concentración de energía espectral), y el ensordecimiento. Sus resultados indican que la producción de /s/ está fuertemente condicionada por la velocidad del habla, la posición de /s/ en la palabra, el contexto fonético, la acentuación silábica y la frecuencia léxica. Específicamente, los autores encontraron más debilitamiento de /s/ cuando los participantes hablan más rápido, 
así como cuando la /s/ aparecía en posición final de palabra o seguida de una vocal que no fuera alta, y en palabras de alta frecuencia. Con este estudio, los investigadores contribuyen significativamente al campo de la fonología al proveer evidencia que apoya tres aspectos importantes: (i) las variables continuas nos permiten examinar patrones acústicos precisos que no se podrían analizar o que incluso pasarían desapercibidos con una metodología categórica; (ii) su análisis ofrece una caracterización detallada del debilitamiento consonántico al contar con múltiples pistas acústicas (i.e., duración, centro de gravedad y ensordecimiento); y (iii) se reduce o se elimina la subjetividad en el análisis, ya que usan medidas acústicas precisas en vez de únicamente la capacidad auditiva del investigador.

Por su parte, Carrasco et al. (2012) y Hualde et al. (2011) ofrecen directrices sobre cómo tomar medidas continuas en la producción de consonantes oclusivas sordas y sonoras. Estos autores proponen tomar medidas basadas tanto en la duración como en la intensidad (p. ej., IntRatio o intensity ratio en inglés). Este tipo de medidas de intensidad indican el grado de oclusión de las consonantes en un continuo que varía desde muy oclusivas hasta muy debilitadas. Carrasco et al. (2012) llevaron a cabo un análisis comparativo de la realización de /b d g/ en posición posconsonántica y posvocálica en Costa Rica y en Madrid. Tradicionalmente se ha aceptado la idea de que cada uno de los fonemas / $\mathrm{b} \mathrm{d} \mathrm{g/consta} \mathrm{de} \mathrm{dos} \mathrm{alófonos}$ que se encuentran en distribución complementaria: por un lado, los alófonos oclusivos [b d g] que aparecen tras pausa, tras nasal, y en el caso de /d/ también después de la lateral /1/; y por otro lado, los alófonos aproximantes [ $\beta$ б y] que aparecen en el resto de contextos (Hualde, 2014). Sin embargo, mediante el uso de medidas acústicas continuas, Carrasco et al. (2012) pudieron proveer evidencia de la existencia de diferencias dialectales entre Costa Rica y Madrid. En Costa Rica, los alófonos oclusivos fueron frecuentes en todos los contextos posconsonánticos y tras deslizadas, y dichas producciones en posición posconsonántica fueron significativamente diferentes a las producciones en posición posvocálica. En Madrid, sin embargo, encontraron un continuo de grados de constricción sin una distinción tan clara entre contextos posvocálicos y posconsonánticos. Por tanto, un análisis continuo de la espirantización de / b d g/ permitió a estos autores observar diferencias dialectales significativas que los análisis categóricos tradicionales no nos habían permitido observar.

Con respecto a las consonantes oclusivas sordas $/ \mathrm{p} \mathrm{t} \mathrm{k/,} \mathrm{a} \mathrm{pesar} \mathrm{de} \mathrm{haberse}$ afirmado tradicionalmente que los fonemas $/ \mathrm{p} \mathrm{t} \mathrm{k/} \mathrm{son} \mathrm{fonéticamente} \mathrm{sordos,}$ Hualde et al. (2011) encontraron que en el habla espontánea se dan casos de /p t $\mathrm{k} /$ producidas con sonoridad total o parcial. El hecho de que las consonantes /p t k/ presenten sonoridad llevó a los autores a cuestionar la posible neutralización de /p t $\mathrm{k} / \mathrm{y} / \mathrm{b} \mathrm{d} \mathrm{g} /$. Para investigar el grado de solapamiento fonético entre estas categorías, los autores usaron medidas de intensidad y duración para examinar sus diferencias o semejanzas. Estos análisis permitieron a los autores descubrir que, aunque tanto $/ \mathrm{p} \mathrm{t} \mathrm{k/} \mathrm{como} \mathrm{/b} \mathrm{d} \mathrm{g/} \mathrm{pueden} \mathrm{producirse} \mathrm{como} \mathrm{variantes} \mathrm{fonéticamente} \mathrm{sonoras,}$ todavía existen diferencias significativas entre $/ \mathrm{p} \mathrm{t} \mathrm{k/} \mathrm{y} \mathrm{/b} \mathrm{d} \mathrm{g/,} \mathrm{ya} \mathrm{que} \mathrm{las} \mathrm{primeras}$ 
suelen ser más largas y más oclusivas que las segundas, por lo que la neutralización y recategorización fonológica en el futuro cercano es algo poco probable. Una vez más, estos hallazgos motivan la realización del presente estudio, puesto que destacan las ventajas de los análisis continuos más recientes frente a análisis categóricos.

Teniendo en cuenta todas estas consideraciones, el objetivo de este artículo será llevar a cabo tanto un análisis continuo como uno categórico, usando los mismos datos y variables, para arrojar luz sobre la idoneidad de cada uno de estos métodos de análisis.

\section{Preguntas de investigación}

Las preguntas que guían esta investigación son las siguientes:

(1) ¿Cuáles son las variables lingüísticas y extralingüísticas que favorecen el debilitamiento de/d/intervocálica cuando dicho debilitamiento es codificado con medidas continuas?

(2) ¿Cuáles son las variables lingüísticas y extralingüísticas que favorecen el debilitamiento de/d/intervocálica cuando dicho debilitamiento es codificado de forma categórica?

(3) En general, ¿cuáles son las ventajas de llevar a cabo análisis continuos y análisis categóricos respectivamente?

\section{Metodología}

\section{Datos y codificación}

Los datos usados para este análisis provienen de entrevistas sociolingüísticas extraídas del corpus PRESEEA (2014). En total, se seleccionaron 16 hablantes con diferentes niveles socioeconómicos, edades y géneros. Específicamente, de los 16 participantes, ocho $(\mathrm{N}=8)$ eran hombres y ocho $(\mathrm{N}=8)$ eran mujeres. Con respecto a la edad, cuatro $(\mathrm{N}=4)$ eran adultos jóvenes de entre 20 y 39 años, seis $(\mathrm{N}$ $=6)$ eran adultos de entre 40 y 59 años, y seis $(\mathrm{N}=6)$ eran adultos de entre 60 y 75 años. Finalmente, cinco $(\mathrm{N}=5)$ participantes pertenecían a la clase social baja, seis $(\mathrm{N}=6)$ pertenecían a la clase media, y otros cinco $(\mathrm{N}=5)$ a la clase alta. Todos los participantes eran originarios de Madrid y hablantes nativos de español.

Se extrajo un total de 965 instancias de /d/ intervocálica dentro de enunciado tanto en posición interior de palabra como en posición inicial de palabra. Para obtener una medida continua del grado de debilitamiento de la /d/, se calculó un índice de intensidad (en adelante IntRatio, abreviatura del inglés intensity ratio). Dicho cálculo fue realizado de manera similar a las formas propuestas por Hualde et al. (2011) y 
Carrasco et al. (2012). Para nuestro propósito, se tomaron medidas de intensidad en tres puntos específicos: en el punto de máxima intensidad en la vocal previa (P1), en el punto de mínima intensidad en la consonante /d/ (V), y en el punto de máxima intensidad de la vocal siguiente (P2). La Figura 1 ilustra estos puntos de los que se tomaron estas medidas en la palabra edad.

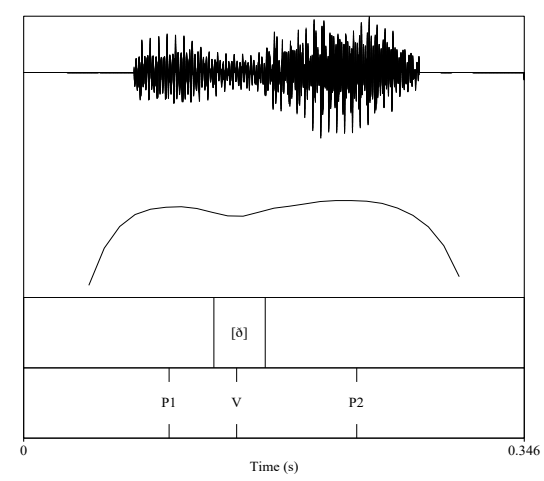

Figura 1. Realización de /d/ intervocálica en la palabra edad y puntos de los que se tomaron medidas de intensidad.

Una vez tomadas las medidas, se dividió el valor de $\mathrm{V}$ (en decibelios) entre el promedio de P1 y P2 (también en decibelios) siguiendo la siguiente fórmula:

$$
\text { IntRatio }=\frac{\mathrm{V} 1(\text { en } \mathrm{dB})}{\text { Promedio de P1 y P2 (en } \mathrm{dB})}
$$

Dicha fórmula proporciona un índice de intensidad para cada instancia de /d/ que oscila entre 0 y 1 . Las producciones con valores más cercanos a 1 son producciones más debilitadas de /d/, mientras que las producciones con un IntRatio más cercano a 0 son producciones más oclusivas. De esta forma, podemos dar cuenta del grado de oclusión o debilitamiento de /d/ de una forma continua. Este IntRatio será la variable dependiente del análisis continuo.

Para el análisis categórico, la producción de /d/ fue codificada como [ð] 0 Ø, es decir, como retención o elisión. Aquellos ítems con un IntRatio igual a 1 fueron considerados elisiones $(\mathrm{N}=132)$, mientras que el resto de ítems con un IntRatio inferior a 1 fueron considerados retenciones $(\mathrm{N}=833)$.

En lo que se refiere a las variables independientes, serán las mismas para ambos análisis e incluirán factores tanto lingüísticos como extralingüísticos, tal y como se describe en las siguientes secciones. 


\section{Variables lingüísticas}

Contexto lingüístico. Se ha demostrado que el contexto lingüístico en el que aparece la/d/intervocálica tiene un efecto significativo en su producción. Por ejemplo, algunas tendencias observadas indican que las vocales $/ 0 / \mathrm{y} / \mathrm{a} /$ suelen favorecer la elisión de /d/, mientras que las vocales altas /i/ y /u/ suelen favorecer la retención (Samper, 2011). Por tanto, se incluirán en el análisis las siguientes dos variables: (i) altura de la vocal anterior (alta, media o baja), y (ii) altura de la siguiente vocal (alta, media o baja). La predicción es que la vocal baja y las medias favorecerán el debilitamiento de /d/, mientras que las altas favorecerán la retención.

Categoría gramatical. Las variantes de esta categoría son: adjetivo, adverbio, participio pasado, pronombre, sustantivo y verbo. Se ha incluido esta variable, ya que los participios pasados y los sustantivos tienden a favorecer la elisión de /d/ intervocálica, mientras que los adjetivos favorecen su retención (Samper, 2011).

Acento silábico. Se espera que cuando la /d/ se encuentre en una sílaba tónica, su producción sea más oclusiva o tienda a retenerse. Por el contrario, si la /d/ se encuentra en sílaba átona, se espera que su realización sea más debilitada.

Posición en la palabra. Esta variable nos indica si la /d/ intervocálica se encuentra en posición inicial o media de palabra. Según estudios previos, se espera que la/d/ sea más debilitada en posición interior de palabra que en posición inicial.

Frecuencia de corpus (log10). Para obtener esta variable, se calculó el logaritmo decimal del número de veces que aparece cada palabra analizada dentro del corpus. Esta variable se ha incluido siguiendo los postulados de la teoría basada en uso (Bybee, 2002, 2006), según los cuales las unidades léxicas más frecuentes experimentarán más reducción y debilitamiento consonántico que los ítems menos frecuentes.

Unidad léxica. Se incluyó como variable al azar en el análisis estadístico.

\section{Variables extralingüísticas}

Género. En este análisis, esta variable incluye dos niveles: hombre y mujer. Esta variable fue incluida, ya que se ha encontrado que los hombres usualmente tienden a usar variantes más vernáculas que las mujeres (véase la Sección 1 de este artículo). Sin embargo, a medida que el fenómeno progresa con el paso del tiempo, esta distinción se hace menos prominente. Por tanto, considerar el género podría informarnos sobre cómo avanza este fenómeno lingüístico en Madrid.

Edad. Esta variable incluye tres niveles: adultos jóvenes (20-39 años), adultos de edad intermedia (40-59 años), y adultos mayores (60-75). Se ha incluido esta variable ya que usualmente es un buen indicador del cambio lingüístico. Por ejemplo, 
si encontráramos que los hablantes jóvenes tienden a producir variantes más reducidas con mayor frecuencia, entonces podríamos estar ante un cambio en curso.

Estatus socioeconómico. Esta variable, que incluye tres niveles (clase baja, clase media y clase alta), se ha incluido porque el debilitamiento de /d/ es un fenómeno motivado por factores sociales, y examinar el efecto de esta variable será informativo para poder conocer el posible grado de prestigio o estigma asociados con este fenómeno.

Hablante. Se incluyó como variable al azar en el análisis estadístico.

\section{Análisis}

Una vez que se codificaron todas estas variables, los datos fueron sometidos a dos análisis estadísticos en R (R Core Team, 2020): un análisis continuo con IntRatio como variable dependiente, y un análisis categórico cuya variable dependiente es binaria (retención o elisión). Para el análisis continuo se llevó a cabo un análisis de regresión lineal de modelo mixto con el paquete lmerTest (Kuznetsova et al., 2017). En dicho análisis se incluyó IntRatio como la variable dependiente, así como nueve efectos fijos (i.e., género, edad, estatus socioeconómico, posición en la palabra, acento silábico, categoría gramatical, frecuencia de corpus, altura de la vocal previa y altura de la vocal siguiente). Finalmente, se incluyeron dos efectos al azar: unidad léxica y hablante.

Se analizó un total de 965 instancias de /d/ intervocálica que fueron extraídas del corpus. La Figura 2 muestra un histograma con la distribución de los datos para el análisis continuo.

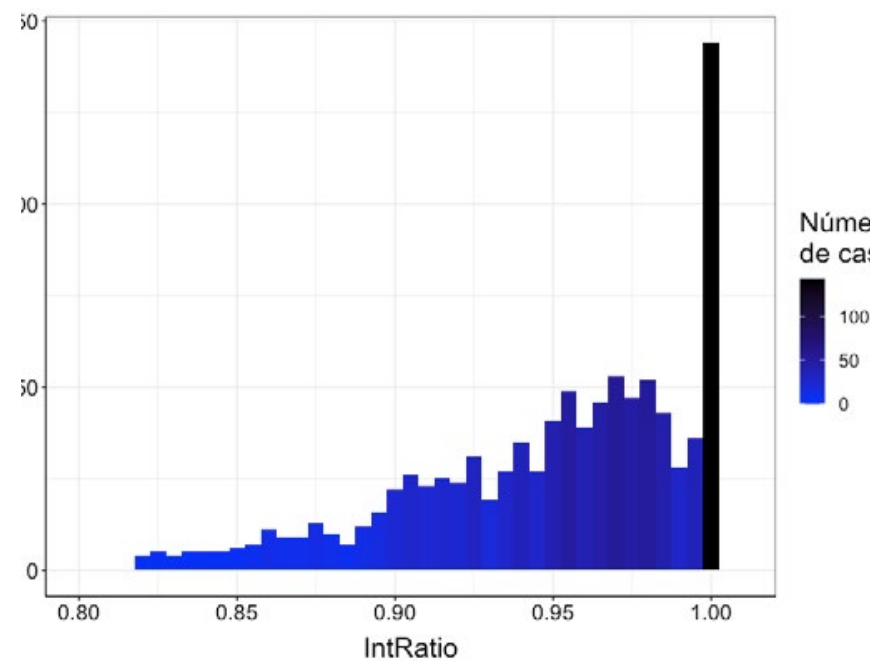

Figura 2. Distribución de los datos según su IntRatio. 
Como se puede observar en la Figura 2, el IntRatio de las producciones de /d/ intervocálica osciló entre 0,82 (la instancia más oclusiva del corpus) y 1 (variantes elididas). Además, se puede notar que la distribución de los datos no es unimodal. Por un lado, esto se debe a que, de las 965 instancias que fueron analizadas, 132 fueron completamente elididas y, por tanto, tienen un IntRatio igual a 1. Por otro lado, la mayoría de los casos de /d/ intervocálica fueron producidos con un IntRatio de entre 0,95 y 1 , lo cual indica que la mayoría de las realizaciones de $/ \mathrm{d} /$, incluso cuando se retuvieron, fueron producidas como variantes muy debilitadas en general. Ante esta distribución de los datos, y con el objetivo de cumplir con los supuestos de regresión lineal (i.e., linealidad, homocedasticidad y normalidad de los residuos), se examinaron los residuos del modelo a través de los gráficos de diagnóstico presentados en la Figura 3.
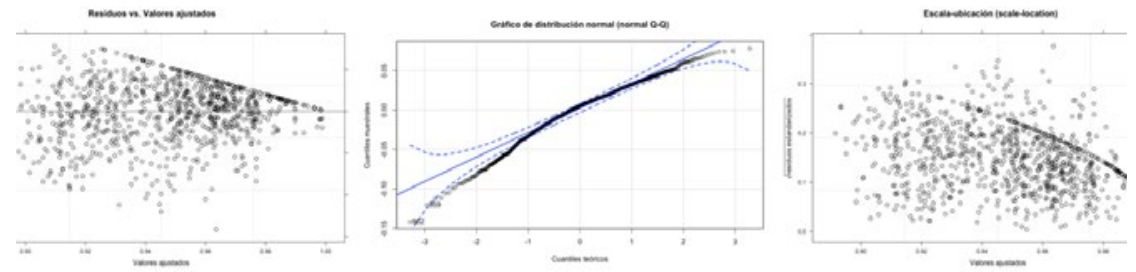

Figura 3. Gráficos de diagnóstico del modelo continuo.

El gráfico del panel de la izquierda representa la distribución de los residuos con respecto a los valores ajustados. En este tipo de gráfico, lo que esperaríamos en un modelo que cumple con los supuestos de regresión lineal sería que los residuos se situaran aleatoriamente alrededor y a lo largo de la línea 0 sin formar un patrón claramente detectable. Esto sugeriría que el supuesto de que la relación es lineal es razonable. Sin embargo, esto no es lo que se observa en la Figura 3, lo cual se debe probablemente al alto número de variantes /d/ con un IntRatio igual a 1.

El panel central de la Figura 3 representa un gráfico de distribución normal. Este tipo de gráficos proporciona una estimación sobre dónde se encuentran los cuantiles muestrales con respecto a los cuantiles teóricos. Una fuerte desviación de la línea proporcionada indicaría que los residuos no están distribuidos de manera normal. En este gráfico en particular se puede observar alguna desviación de la línea normal esperada hacia las colas, pero en general la línea parece recta.

A continuación, el gráfico del panel de la derecha en la Figura 3 nos muestra si los residuos se distribuyen por igual a lo largo de los rangos de los predictores. En este caso, se cumple el supuesto de homogeneidad de varianza si se observa una distribución homogénea y aleatoria de los datos sin la presencia de algún patrón aparente. En nuestro caso, se observa que la variabilidad de los residuos disminuye significativamente a medida que aumenta el valor ajustado. 
Tras examinar estos gráficos se determinó que el modelo no cumple con todos los supuestos del modelo de regresión lineal. Como se mencionó anteriormente, es posible que esto se deba al alto número de casos cuyo IntRatio es igual a 1. Por tanto, para garantizar que se cumple con los supuestos del modelo, se eliminaron todas las instancias de /d/ cuyo IntRatio es igual a 1 , y se volvieron a analizar los gráficos de diagnóstico. Al examinar de nuevo dichos gráficos se observó una mejora considerable con respecto a los supuestos del modelo de regresión lineal. Por tanto, para el análisis continuo se usaron los datos de IntRatio excluyendo aquellos ítems cuyo valor era igual a 1 (i.e., instancias totalmente elididas).

Finalmente, también se revisó la ausencia de multicolinealidad. En análisis de regresión múltiple, multicolinealidad hace referencia a modelos en los que más de dos variables independientes están correlacionadas. Puesto que la presencia de multicolinealidad constituiría un problema que afectaría negativamente el rendimiento de un modelo de regresión, es importante asegurar la ausencia de multicolinealidad en nuestro modelo. Esto se puede evaluar calculando lo que se conoce como Factor de Inflación de Varianza (VIF, por sus siglas en inglés) que, como su propio nombre indica, mide cuánto se infla la varianza de un coeficiente de regresión debido a la multicolinealidad en el modelo. El menor valor posible es 1 (ausencia de multicolinealidad). Como regla general, un valor de VIF que excede 50 10 indica que hay un problema de multicolinealidad (James et al., 2013). En nuestro estudio se calculó el VIF de cada variable independiente usando el paquete car (Fox \& Weisberg, 2019) en R (R Core Team, 2020). Los resultados mostraron que el VIF de las variables dependientes osciló entre 1.01 para la variable de género y 2.86 para la variable de categoría gramatical, lo cual indica ausencia de multicolinealidad en el modelo de regresión.

Con respecto al análisis categórico, se llevó a cabo un análisis de regresión logística de modelo mixto usando el paquete lme4 (Bates et al., 2015). La variable dependiente fue la realización binaria de /d/ (i.e., retención o elisión). En este análisis se incluyeron los mismos efectos fijos y aleatorios que en el análisis continuo. También se llevó a cabo una revisión de las variables independientes y se confirmó que no había multicolinealidad en este modelo.

\section{Resultados}

\section{Análisis continuo}

El análisis de regresión lineal de modelo mixto reveló un efecto significativo de cinco variables: la edad, el acento silábico, la categoría gramatical y la frecuencia de corpus. La Figura 4 ilustra de forma gráfica el efecto de las variables significativas con respecto al IntRatio. Los coeficientes específicos del análisis de regresión aparecen en el Apéndice A. 

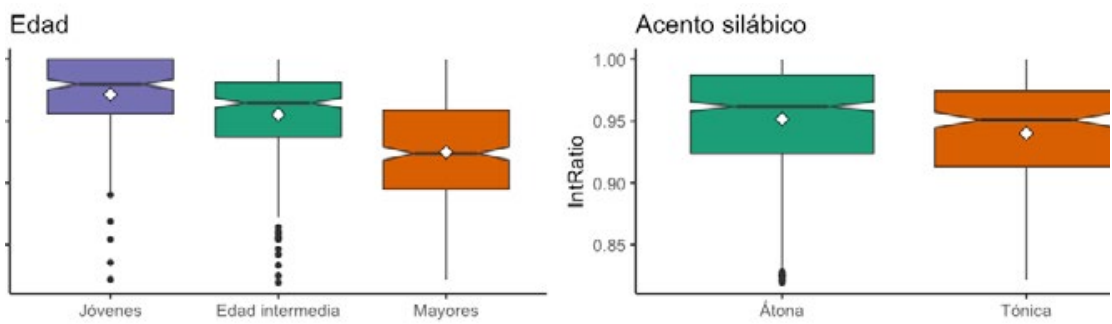

Categoria gramatical
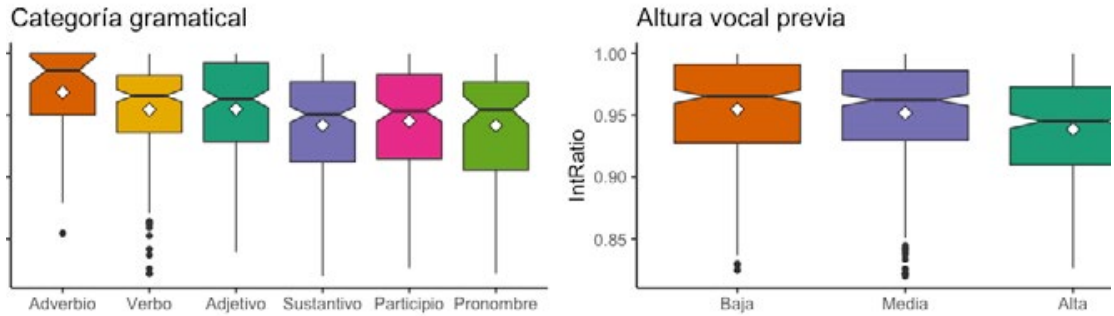

Frecuencia de corpus

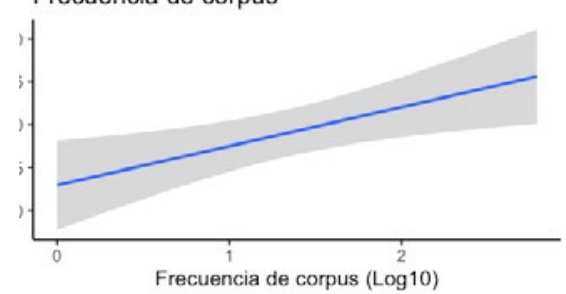

Figura 4. Variables significativas en el análisis continuo.

Con respecto a la edad se encontró que los hablantes más jóvenes constituyen el grupo que produce variantes de /d/ más reducidas (i.e., mayor IntRatio), mientras que el grupo de hablantes de mayor edad producen variantes más reforzadas.

En cuanto a las variables lingüísticas, el análisis reveló que hay más debilitamiento de /d/ en sílabas átonas que en sílabas tónicas. Asimismo, se encontró que hay más debilitamiento de /d/ cuando la vocal previa es baja o media que cuando dicha vocal es alta. Estos resultados apoyan los hallazgos de estudios previos que se resumieron en la Sección 1 (Samper, 2011). En cuanto a la categoría gramatical, curiosamente se halló que los adverbios y los verbos son las categorías gramaticales que más favorecen el debilitamiento, mientras que los pronombres y los participios favorecen producciones más reforzadas. Finalmente, se observó el efecto esperado de la frecuencia de corpus: a medida que aumenta la frecuenta, aumenta el IntRatio. Es decir, hay más debilitamiento consonántico a medida que la frecuencia léxica aumenta. 


\section{Análisis categórico}

Con respecto al análisis categórico, la regresión logística sugiere que tres variables tienen un efecto significativo en la elisión de /d/ intervocálica. Estas variables son la edad, la altura de la vocal previa y la categoría gramatical. La Figura 5 muestra de forma gráfica el efecto estas variables en la elisión de /d/. Los coeficientes de la regresión logística aparecen en el Apéndice B.
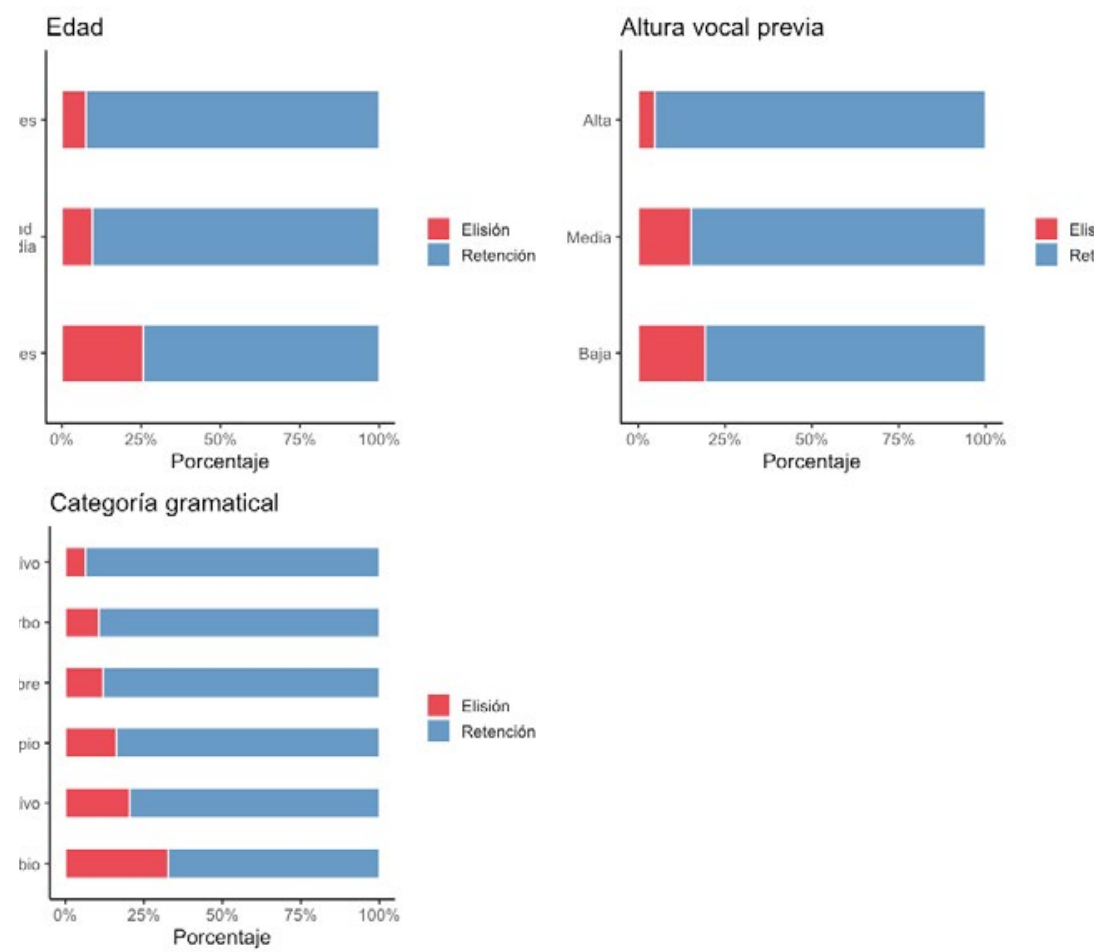

Figura 5. Variables significativas en el análisis categórico.

Como se puede observar, con respecto a la edad, el análisis reveló que hay más elisión de /d/ intervocálica entre los hablantes más jóvenes, mientras que hubo más retención entre los hablantes mayores.

En cuanto a las variables lingüísticas se encontró que hay más casos de elisión cuando la vocal anterior es baja, mientras que hubo mayor retención cuando la vocal previa era una vocal alta. Finalmente, se hallaron diferentes tasas de elisión al comparar diferentes categorías gramaticales. En este caso, los adverbios mostraron una mayor tasa de elisión, seguidos de los adjetivos y los participios. Por el contrario, los sustantivos constituyen la categoría gramatical con la menor tasa de elisión. 
En resumen, tanto el análisis categórico como el continuo sugieren que el debilitamiento de /d/ intervocálica está condicionado por factores tanto lingüísticos como extralingüísticos. Aunque se observaron algunas semejanzas en los resultados de estos análisis, también se encontraron algunas diferencias al comparar los resultados de ambos análisis. En la siguiente sección se ofrece una discusión de estos resultados y sus implicaciones para los análisis sociofonéticos.

\section{Discusión}

En las dos primeras preguntas de investigación que motivaron la realización de este análisis nos preguntábamos cuáles son los factores lingüísticos y extralingüísticos significativos que condicionan la producción de /d/ intervocálica. También nos preguntábamos cómo se comparan los resultados de un análisis continuo y un análisis categórico. Como se refleja en la sección previa, se encontraron varias semejanzas, así como también algunas diferencias en los resultados de dichos análisis. Con respecto a las semejanzas encontradas, destacamos el efecto de la edad. Tanto en el análisis categórico como en el continuo fueron los hablantes más jóvenes los que favorecieron el debilitamiento o la elisión total de /d/, mientras que los hablantes mayores favorecían su retención. La segunda semejanza hallada tiene que ver con el efecto de la vocal previa. Ambos análisis revelaron que las vocales altas favorecen el debilitamiento, mientras que la vocal baja y las vocales medias favorecen la retención de /d/ intervocálica.

Aunque se encontraron efectos significativos en ambos análisis con respecto a la categoría gramatical, se pudieron observar algunas diferencias al comparar ambos modelos. Por ejemplo, en el análisis continuo, las categorías gramaticales pueden ordenarse de la siguiente manera: adverbios $>$ verbos $>$ adjetivos $>$ sustantivos $>$ participios $>$ pronombres, y los adverbios fueron los que favorecieron mayor debilitamiento y los pronombres los que favorecieron más reforzamiento. En cambio, en el análisis categórico, las categorías gramaticales seguían el siguiente orden según su tasa de elisión: adverbios $>$ adjetivos $>$ participios $>$ pronombres $>$ verbos $>$ sustantivos, y los adverbios fueron la categoría con la tasa de elisión más alta, y los pronombres la categoría con la tasa de elisión más baja.

Además de estas semejanzas y diferencias, también es importante destacar dos variables independientes que fueron significativas en el análisis continuo, pero no en el categórico: el acento silábico y la frecuencia de corpus. Específicamente, el modelo continuo reveló que las sílabas átonas favorecen el debilitamiento, y que el debilitamiento de /d/ aumenta a medida que aumenta la frecuencia de corpus.

Ante estos hallazgos, en este artículo se argumenta que las principales diferencias encontradas al comparar los dos análisis tienen que ver con el hecho de que mientras que un análisis continuo da cuenta de la naturaleza gradual de los procesos de debilitamiento consonántico, un modelo categórico solamente da cuenta del caso más extremo de reducción: la elisión. Por consiguiente, no sorprende que existan algunas diferencias en los resultados al comparar los dos análisis. Así, la 
selección de uno u otro modelo (o ambos) deberá estar motivada por las preguntas de investigación que el investigador plantee.

En esta misma línea, en la tercera pregunta de investigación propuesta en el presente estudio nos preguntábamos cuáles serían las posibles ventajas de llevar a cabo un análisis con medidas continuas frente a uno categórico y viceversa. A lo largo de este artículo se han mencionado algunas de estas ventajas de efectuar un análisis continuo. Por ejemplo, la subjetividad a la hora de codificar la producción de /d/ intervocálica se ve reducida de manera significativa al tomar medidas continuas en comparación con codificaciones impresionistas basadas principalmente en la audición del investigador, ya que el modelo continuo incluye medidas acústicas precisas de las propiedades físicas de los sonidos (p. ej., IntRatio en nuestro caso). Además, un análisis continuo proporciona detalles y matices relacionados con la variación y puede explicar el proceso completo de la lenición o debilitamiento consonántico. Por ejemplo, una producción de /d/ puede ser retenida, pero su articulación específica puede variar desde una producción muy oclusiva a una muy reducida. Un análisis categórico no refleja esta variación tan natural y propia de los procesos de debilitamiento consonántico. No obstante, como se argumentó como se argumentó en secciones previas de este artículo, un análisis de los gráficos de diagnóstico del modelo continuo reveló que los datos acústicos de IntRatio no cumplían con todos los supuestos para el modelo de regresión continua, principalmente por la alta presencia de ítems léxicos cuyo IntRatio era igual a 1 (i.e., elisiones). Así, con el objetivo de cumplir con los supuestos para el análisis de regresión lineal, se decidió eliminar estos casos del análisis continuo. De esta manera, aunque el modelo continuo da cuenta de la gradualidad del debilitamiento consonántico, no lo hace de los casos más extremos de debilitamiento, es decir, los casos de elisión. Por el contrario, aunque el modelo categórico no informa de la gradualidad del debilitamiento consonántico, sí informa precisamente de la elisión total. Por estas razones, los hallazgos presentados en este análisis sugieren que al examinar un fenómeno sociofonético el investigador puede beneficiarse de la combinación de un análisis continuo con uno categórico.

\section{Conclusión}

En este artículo se ha llevado a cabo una comparación de dos modelos de análisis sociofonético sobre el debilitamiento consonántico de la /d/ intervocálica en Madrid. En el primer modelo, el debilitamiento de /d/ ha sido conceptualizado de manera continua. Para ello, el grado de oclusión o debilitamiento fue cuantificado tomando medidas acústicas y creando un índice de intensidad (IntRatio). En el segundo análisis, el debilitamiento de /d/ fue clasificado en dos categorías: retención y elisión total. El objetivo principal de la investigación fue comparar los resultados obtenidos en los dos modelos y discutir algunas de las ventajas o limitaciones de estos.

Los resultados de la investigación sugieren que, aunque los efectos de algunas de las variables independientes fueron similares en ambos análisis, otros fueron diferentes. Por tanto, se ha propuesto que estas diferencias se deben a la naturaleza 
diferente de cada análisis. Mientras que un análisis continuo da razón de una amplia gama de posibles realizaciones (i.e., desde producciones reforzadas y oclusivas, hasta variantes muy debilitadas), el análisis categórico solamente reporta las producciones elididas, pero no de todo el espectro de posibles realizaciones. Por tanto, se ha argumentado que la selección de uno u otro análisis deberá estar justificada y dependerá de los propósitos del investigador, aunque se ha sugerido que las investigaciones sociofonéticas pueden beneficiarse de forma significativa de la combinación de análisis continuos con análisis categóricos, puesto que dicha combinación (i) nos permitirá llevar a cabo investigaciones en las que la subjetividad en la codificación se vea reducida, (ii) arrojará luz sobre la gradualidad que caracteriza el debilitamiento consonántico, (iii) dará cuenta de toda la gama de posibles realizaciones, y (iv) se cumplirá con los supuestos para los diferentes análisis de regresión.

\section{Referencias}

Bates D., Mächler M., Bolker B., \& Walker S. (2015). Fitting Linear Mixed-Effects Models Using Ime4. Journal of Statistical Software, 67(1), 1-48. https://doi. org/10.18637/jss.v067.i01.

Blanco, A. (2004). Estudio sociolingüístico de Alcalá de Henares. Servicio de Publicaciones de la Universidad de Alcalá.

Bybee, J. (2002). Word Frequency and Context of Use in the Lexical Diffusion of Phonetically Conditioned Sound Change. Language Variation and Change 14(3), 261-290. https://doi.org/10.1017/S0954394502143018.

Bybee, J. (2006). From Usage to Grammar: The Mind's Response to Repetition. Language, 82(4), 711-733. https://doi.org/10.1353/lan.2006.0186.

Carrasco, P., Hualde, J. I., \& Simonet, M. (2012). Dialectal Differences in Spanish Voiced Obstruent Allophony: Costa Rican versus Iberian Spanish. Phonetica, 69(3), 149-179. https://doi.org/10.1353/lan.2006.018610.1159/000345199.

Díaz-Campos, M. (2016). Nuevas tendencias en el análisis de la variación sociofonológica: una comparación de análisis tradicionales basados en la categorización en contraste con estudios fundamentados en medidas acústicas. En Ponencia presentada en el 8th International Workshop on Spanish Sociolinguistics. Universidad de Puerto Rico.

Díaz-Campos, M., Scrivner, O., \& Delgado-Díaz, G. (2015). A Real Time Comparison of the Lenition of Intervocalic /d/ in Spanish: Examining the Changes in the Sociolinguisic Profile from 1997 to 2004-2010. En Póster presentado en NWAV44 (New Ways of Analyzing Variation 44). Universidad de Toronto. 
File-Muriel, R. J., \& Brown, E. K. (2011). The Gradient Nature of S-Lenition in Caleño Spanish. Language Variation and Change, 23(02), 223-243. https://doi. org/10.1017/S0954394511000056.

Fox, J., \& Weisberg, S. (2019). An R Companion to Applied Regression (3rd ed.). Sage.

Gil, N. (2004). Estudio sociolingüístico de la fonética de la ciudad de Madrid. (Memoria de licenciatura inédita). Universidad de Alcalá.

Gómez, A. (1994). Aspectos del habla de Linares (Jaén). (Tesis doctoral). Universidad de Málaga.

Gómez, J. R., \& Gómez, M. (2010). Mantenimiento y elisión de la /d/ intervocálica en el español de Valencia. Verba, 37, 89-122.

Hualde, J. I., (2014). Los sonidos del español. Cambridge University Press.

Hualde, J. I., Simonet, M., \& Nadeu, M. (2011). Consonant Lenition and Phonological Recategorization. Laboratory Phonology, 2(2), 301-329. https:/doi. org/10.1515/labphon.2011.011.

James, G., Witten, D., Hastie, T., \& Tibshirani, R. (2013). An Introduction to Statistical Learning: With Applications in R. Springer.

Jiménez, R. (2020). Variación fonológica de la/d/intervocálica en el sociolecto alto de Sevilla. Rilce, 36(2), 674-707. https://doi.org/10.15581/008.36.2.674-707.

Kuznetsova, A., Brockhoff, P., \& Christensen, R. (2017). ImerTest Package: Tests in Linear Mixed Effects Models. Journal of Statistical Software, 82(13), 1-26. https://doi.org/10.18637/jss.v082.i13.

Lozano, M. C. (1979). Stop and Spirant Alternations: Fortition and Spirantization Processes in Spanish Phonology. (Tesis doctoral). Indiana University.

Malaver, I. \& Perdomo, L. (2016). La elisión de /d/ en posición intervocálica en la comunidad caraqueña. Boletín de Filología, 51(2), 147-179.

Mascaró, J. (1991). Iberian Spirantization and Continuant Spreading. Catalan Working Papers in Linguistics, 1, 167-179.

Moya, J. A. (1979). La pronunciación del español en Jaén. (Tesis doctoral). Universidad de Granada.

Moya, J. A. (2009). Las tensiones del cambio lingüístico a propósito de la elisión de la /d/ intervocálica. En E. Montoro del Arco (ed.), El español del siglo XXI. 
Actas de las XIV Jornadas sobre la lengua española y su enseñanza (pp. 211-220). Universidad de Granada.

Ortega-Llevaria, M. (2003). Effects of Phonetic and Inventory Constraints in the Spirantization of Intervocalic Voiced Stops: Comparing Two Different Measurements of Energy Change. In M. J. Solé, D. Recasens \& J. Romero (eds.), Proceedings of the XV International Congress of Phonetic Sciences (pp. 2817-2820).

Pérez , A. M. (2003). Estudio sociolingüístico de El Hierro. (Tesis doctoral). Universidad de Las Palmas de Gran Canaria.

PRESEEA (2014). Corpus del Proyecto para el estudio sociolingüístico del español de España y de América. Universidad de Alcalá. http://preseea.linguas.net.

R Core Team (2020). R: A Language and Environment for Statistical Computing. R Foundation for Statistical Computing, Vienna, Austria. https:/www.R-project. org/.

Rogers, B. (2016). The Influence of Linguistic and Social Variables in the Spirantization of Intervocalic /b, d, g/ in Concepción, Chile. Studies in Hispanic and Lusophone linguistics, 9(1), 207-237. https://doi.org/10.1515/shll-20160008.

Samper, J. A. (2011). Sociophonological Variation and Change in Spain. In M. DíazCampos (ed.), The Handbook of Hispanic Sociolinguistics (pp. 98-120). Wiley-Blackwell. 


\section{Apendice A}

\begin{tabular}{llrrrr}
\hline \multicolumn{2}{l}{ Tabla A. Resultados de la regresión lineal } & & & & \\
\hline Variables & Comparación & Coef. & Error est. & $\boldsymbol{t}$ & $\boldsymbol{p}$ \\
\hline (Intersección) & -- & 0.934 & 0.008 & 111.693 & $<0.001$ \\
\hline Género (Ref.: Mujer) & Hombre & 0.008 & 0.004 & 1.988 & n.s. $^{{ }^{2}}$ \\
\hline Edad (Ref.: Intermedia) & Mayores & -0.03 & 0.005 & -6.274 & $<0.001$ \\
& Jóvenes & 0.01 & 0.006 & 2.334 & $<0.05$ \\
\hline Clase (Ref.: Baja) & Media & 0.003 & 0.006 & 0.574 & n.s. \\
& Alta & -0.003 & 0.005 & -0.594 & n.s. \\
\hline Posición (Ref.: Interior) & Media & -0.001 & 0.005 & -0.008 & n.s. \\
\hline Acento (Ref.: Átona) & Tónica & -0.01 & 0.003 & -3.015 & $<0.01$ \\
\hline Categoría (Ref.: Adj.) & Adverbio & 0.009 & 0.007 & 1.342 & n.s. \\
& Sustantivo & -0.002 & 0.004 & -0.550 & n.s. \\
& Participio & 0.002 & 0.005 & 0.480 & n.s. \\
& Pronombre & -0.016 & 0.006 & -2.557 & $<0.05$ \\
& Verbo & 0.011 & 0.005 & 2.208 & $<0.05$ \\
\hline Vocal siguiente & Baja & 0.007 & 0.004 & 1.759 & n.s. \\
(Ref.: Alta) & Media & -0.001 & 0.004 & -0.092 & n.s. \\
\hline Vocal previa & Baja & 0.008 & 0.003 & 2.441 & $<0.05$ \\
(Ref.: Alta) & Media & 0.006 & 0.004 & 1.639 & n.s. \\
\hline Frecuencia léxica & --- & 0.005 & 0.002 & 2.348 & $<0.05$ \\
\hline${ }^{a}$ n.s. = no significativo & & & & &
\end{tabular}

\section{Apéndice B}

\begin{tabular}{llrrrr}
\hline \multicolumn{2}{l}{ Tabla B. Resultados de la regresión logística } & & & & \\
\hline Variables & Comparación & Coef. & Error est. & $\boldsymbol{z}$ & $\boldsymbol{p}$ \\
\hline (Intersección) & -- & 4.121 & 1.066 & 3.868 & $<0.001$ \\
\hline Género (Ref.: Mujer) & Hombre & 0.126 & 0.248 & 0.510 & n.s. $^{{ }^{2}}$ \\
\hline Edad (Ref.: Intermedia) & Mayores & 0.477 & 0.343 & 1.391 & n.s. \\
& Jóvenes & -1.327 & 0.335 & -3.959 & $<0.001$ \\
\hline Clase (Ref.: Baja) & Media & 0.337 & 0.364 & 0.924 & n.s. \\
& Alta & 0.526 & 0.274 & 1.920 & n.s. \\
\hline Posición (Ref.: Interior) & Media & -0.362 & 0.489 & -0.739 & n.s. \\
\hline Acento (Ref.: Átona) & Tónica & 0.666 & 0.480 & 1.389 & n.s. \\
\hline Categoría (Ref.: Adj.) & Adverbio & -0.571 & 0.679 & -0.840 & n.s. \\
& Sustantivo & 0.761 & 0.480 & 1.585 & n.s. \\
& Participio & -0.594 & 0.539 & -1.101 & n.s. \\
& Pronombre & 1.098 & 0.521 & 2.109 & $<0.05$ \\
& Verbo & 0.574 & 0.613 & 0.936 & n.s. \\
\hline Vocal siguiente & Baja & 0.571 & 0.563 & 1.013 & n.s. \\
(Ref.: Alta) & Media & -0.046 & 0.486 & -0.095 & n.s. \\
\hline Vocal previa & Baja & -1.714 & 0.488 & -3.513 & $<0.001$ \\
(Ref.: Alta) & Media & -1.479 & 0.541 & -2.736 & $<0.01$ \\
\hline Frecuencia léxica & -- & -0.372 & 0.242 & -1.542 & n.s. \\
\hline${ }^{a}$ n.s. = no significativo & & & & & \\
\hline
\end{tabular}

